\title{
Mushy Layer Formation during Solidification of Binary Alloys from a Cooled Wall: the Role of Boundary Conditions
}

\author{
I.V. Alexandrova, D.V. Alexandrov, D.L. Aseev and S.V. Bulitcheva \\ Department of Mathematical Physics, Urals State University \\ Ekaterinburg 620083, Russian Federation
}

\begin{abstract}
Motivated by metallurgical and geophysical applications we present a mathematical model describing the process of unidirectional solidification of binary melts or solutions, which is based on the classical Stefan thermodiffusion theory with a planar front up to a time of mushy layer initiation and on the quasi-equilibrium theory of solidification with a constitutionally supercooled region afterwards. The role of boundary conditions imposed at the cooled wall is studied in terms of heat flux coefficients describing ultimately nonlinear dynamics of the process.
\end{abstract}

PACS numbers: 44.05.+e, 81.30.Fb

\section{Introduction}

Aspects of forming of various types of micro- and macrostructures in solids and liquids, the physical mechanisms of which remain to a large degree unclear, are of particular importance. Directional and bulk crystallization of liquids underlies many technologies employed in traditional and new industries (metallurgy, energetics, aerospace engineering, electronics) and describes natural phenomena (formation of ices, solidification of lava-streams, crystal growth in supersaturated solutions). In spite of the extended history of study of crystallization, many aspects of the physics of this phenomenon remain unclear.

Traditionally, the study of crystallization was and is performed within the framework of the classical model, leading to the Stefan boundary value problem. In this approach it is assumed that the liquid and solid phases are separated by a clearly expressed smooth (planar, cylindrical, spherical, etc.) interface between the phases, heat transfer occurs by conduction according to the Fourier law and the velocity of the crystallization front is controlled by the absorption of heat by the solid phase. The mathematical formulations corresponding to these physical models belong to the class of highly-nonlinear problems with moving boundaries. In spite of the appreciable progress attained in investigating these problems, it became clear during the past several years that this approach is limited.

A number of important contributions to the study of these problems has been made previously. Ivantsov [1] demonstrated that, under certain conditions, a region of impurity-induced (constitutional) supercooling i.e., one in which the temperature is lower than the temperature of the phase transition, forms in the melt. If such a region is absent, a solidification process is to be studied in a standard way on the basis of the well-known Stefan problem.

If it exists, the situation changes rather drastically as a result of originating of a two-phase zone containing solid phase elements in the form of either dendrites or newly born crystals suspended in the ambient liquid. The structure of this mushy layer depends, first of all, upon a relation between the kinetics of both the solid phase formation and the front motion. When the former one is much slower than the latter, the mushy region is almost free from solid elements and the Stefan (frontal) problem provides for a sufficiently good approximation.

In the opposite limiting case, the two-phase layer structure is nearly equilibrium so that its local temperature coincides with that of the phase transition at a given point. This property permits a special heat and mass transfer problem to be formulated throughout the mushy region without accounting for the kinetics of the new phase formation process [2-4]. This paper is devoted to the intermediate stage of the process when the classical Stefan scenario with a planar front changes by another one with a growing mushy layer.

\section{The model}

Let us consider a unidirectional solidification process generated by the external cooling of the solid wall $x=0$. The regions $0<x<X(t)$ and $X(t)<x<L$ filled with a liquid and solid material are separated by an interface $X(t)$ of zero thickness moving with time $t$ and representing actual crystallization front. To simplify the matter in order to leave main ideas unencumbered with un- 
necessary details, we assume without further comments that: (1) the convective solute transfer (and, in particular, that due to a possible difference in the solid and liquid phase densities) is negligible; (2) a relevant part of the melt phase diagram is composed of straight liquidus and solidus lines; and (3) all thermophysical parameters do not depend on the temperature and the solute concentration.

Initially, there is a thin veneer of solid phase near the boundary $x=0$ so that $X(0)=\varepsilon L$, where $\varepsilon \ll 1$ is a given parameter. In each region the heat and mass conduction equations hold true (diffusion in the solid phase is traditionally negligible)

$$
\begin{aligned}
& \frac{\partial T_{\mathrm{S}}}{\partial t}=a_{\mathrm{S}} \frac{\partial^{2} T_{\mathrm{S}}}{\partial x^{2}}, \quad 0<x<X(t), \\
& \frac{\partial T_{\mathrm{L}}}{\partial t}=a_{\mathrm{L}} \frac{\partial^{2} T_{\mathrm{L}}}{\partial x^{2}}, \quad \frac{\partial C_{\mathrm{L}}}{\partial t}=D_{\mathrm{L}} \frac{\partial^{2} C_{\mathrm{L}}}{\partial x^{2}}, \\
& X(t)<x<L,
\end{aligned}
$$

where $T$ and $C$ are the temperature and impurity concentration fields, $a$ and $D$ are the temperature diffusivity and diffusion coefficient, respectively (subscripts S and L designate the solid and liquid phases). Boundary conditions imposed at the solid-liquid interface $x=X(t)$ can be written in the form [5]:

$$
\begin{aligned}
& T_{\mathrm{S}}=T_{\mathrm{L}}, \quad k_{\mathrm{S}} \frac{\partial T_{\mathrm{S}}}{\partial x}-k_{\mathrm{L}} \frac{\partial T_{\mathrm{L}}}{\partial x}=L_{V} \frac{\mathrm{d} X}{\mathrm{~d} t}, \\
& T_{\mathrm{L}}=T_{0}-m C_{\mathrm{L}}, \quad x=X(t), \\
& (1-k) C_{\mathrm{L}} \frac{\mathrm{d} X}{\mathrm{~d} t}+D_{\mathrm{L}} \frac{\partial C_{\mathrm{L}}}{\partial x}=0, \quad x=X(t),
\end{aligned}
$$

where $k$ is the thermal conductivity coefficient, $L_{V}$ is the latent heat parameter, $T_{0}$ is the temperature of pure substance, $m$ is the liquidus slope, and $k$ is the partition coefficient equal to the ratio of concentrations in the solid $\left(C_{\mathrm{S}}\right)$ and liquid $\left(C_{\mathrm{L}}\right)$ phases at the front (this expression enables us to find the concentration in solid at $x=X(t)$ and, thus the concentration profile in the solid phase region $0<x<X(t)$ as a prehistory of the front motion). Boundary conditions (3) and (4) represent continuity equation, heat balance equation, liquidus equation and mass balance equation.

Let us now consider the case of active cooling of the solid boundary $x=0$, which can be attained, for example, by means of a fluid that bathes this wall. The latter can be expressed by the boundary condition of the form

$$
k_{\mathrm{S}} \frac{\partial T_{\mathrm{S}}}{\partial x}-k_{\mathrm{L}} g_{\mathrm{L}}=\alpha_{a} t, \quad x=0,
$$

where $g_{\mathrm{L}}$ is the temperature gradient in the liquid at $x=L$ and $\alpha_{a}$ is the cooling coefficient. At the opposite solid wall $x=L$, the temperature and concentration gradients are fixed

$$
\frac{\partial T_{\mathrm{L}}}{\partial x}=g_{\mathrm{L}}, \quad \frac{\partial C_{\mathrm{L}}}{\partial x}=0, \quad x=L,
$$

The last condition expresses the fact of impenetrability of an ingot mould. Let at $t=0$ the impurity concentration is equal to the concentration far from the front at the wall $x=L$ :

$$
C_{\mathrm{L}}=C_{\mathrm{L} \infty}, \quad t=0,
$$

and the temperature profiles are linear functions of $x$ :

$$
\begin{aligned}
& T_{\mathrm{L}}=T_{0}-m C_{\mathrm{L} \infty}+g_{\mathrm{L}}[x-X(0)], \\
& T_{\mathrm{S}}=T_{0}-m C_{\mathrm{L} \infty}+\frac{k_{\mathrm{L}}}{k_{\mathrm{S}}} g_{\mathrm{L}}[x-X(0)], \quad t=0 .
\end{aligned}
$$

The last conditions seem quite natural due to the fact that the front rate $\mathrm{d} X / \mathrm{d} t=0$ at $t=0$.

Under certain conditions at $t=t_{*}$ a mushy region can emerge ahead of the front, in which case the Stefan thermodiffusion model described above becomes inapplicable. In the proximity of the moving solidification interface a redistribution of impurity takes place and the melt temperature is found to be below that of the liquidus $T_{\mathrm{L}}<T_{0}-m C_{\mathrm{L}}$. This constitutional supercooling disappears in the mushy region. Here we consider a simplest model of a narrow equilibrium mushy layer formulated in [5] for $t>t_{*}$. Mathematically, this regime is described by modified boundary condition, that is, instead of the mass balance boundary condition (4), we have

$$
-m \frac{\partial C_{\mathrm{L}}}{\partial x}=\frac{\partial T_{\mathrm{L}}}{\partial x}, \quad x=X(t) .
$$

This relationship determines impurity distribution under conditions of a narrow equilibrium mushy region. Thus, simulation of crystallization, taking into account the mushy layer, is connected with changeover from conditions (1)-(8) to conditions (1)-(3) and (5)-(9). The criterion for the emergence of a mushy layer is the inequality

$$
\frac{\partial T_{\mathrm{L}}}{\partial x} \leq-m \frac{\partial C_{\mathrm{L}}}{\partial x}, \quad x=X(t) .
$$

Thus, we come to the problem with a changeover of boundary conditions: if inequality (10) does not hold, then conditions (1)-(8) are used, otherwise conditions (1) $-(3)$ and (5)-(9).

\section{Results and discussions}

Difference methods [6] are used for an approximate solution of the above problem. The main special feature of the posed problem is the presence of a free boundary $x=X(t)$. For such one-dimensional problems most effective are computational algorithms based on a changeover to new variables in which this phase transition interface is fixed (front rectification methods). The calculations were carried out on a sufficiently detailed grid with a total number of sites in the solid and liquid phases equal to 200. In the computation examples given below the following values of physical parameters for the actual $\mathrm{Fe}-\mathrm{Ni}$ alloy were used: $k=0.68, T_{0}=1529.5^{\circ} \mathrm{C}, m=2.65^{\circ} \mathrm{C} /$ wt. $\%$, $L_{V}=3398.5 \mathrm{cal} / \mathrm{cm}^{3}, D_{\mathrm{L}}=5 \times 10^{-5} \mathrm{~cm}^{2} / \mathrm{s}$,

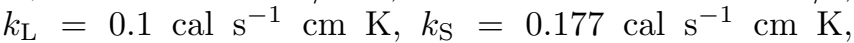
$a_{\mathrm{L}}=0.14 \mathrm{~cm}^{2} / \mathrm{s}, a_{\mathrm{S}}=0.25 \mathrm{~cm}^{2} / \mathrm{s}, C_{\mathrm{L} \infty}=0.3 \mathrm{wt} . \%$, $g_{\mathrm{L}}=10^{\circ} \mathrm{C} / \mathrm{cm}, \varepsilon=0.01, \alpha_{a}=0.02 \mathrm{cal} \mathrm{s}^{-2} \mathrm{~cm}^{2}$.

Figures 1-3 show that the impurity concentration in the solid phase attains its maximum at the time of mushy layer initiation. The reason of this phenomenon can be explained as follows. The liquid phase (melt or solution) solidifies in accordance with the classical Stefan ther- 
modiffusion model up to the point of the emergence of a mushy layer so that the front of solidification moving towards the melt displaces impurity inclusions $(k<1)$. In other words, the impurity concentration at the front on the melt side becomes greater than the impurity concentration at the solid wall $x=L$.

After the emergence of a mushy layer, solid phase elements grow in the ambient liquid (mushy region), where the impurity concentration is smaller than the melt concentration at the interface solid phase-mushy layer. After a while, these solid elements will be absorbed by the moving interface and, as a consequence, the impurity concentration in the solid will be smaller than this concentration up to the moment of mushy layer initiation.

Figures 1-3 also demonstrate that the temperature distributions in both the phases are linear functions of the spatial coordinate. The latter can be explained in terms of the estimates for relaxation times. We should focus our attention on the fact that relaxation times of the temperature fields in the liquid and solid phases, $l^{2} / a_{\mathrm{L}}$ and $l^{2} / a_{\mathrm{S}}$, are a few orders of magnitude smaller than relaxation time $l^{2} / D_{\mathrm{L}}$ of solute diffusion within the liquid phase ( $l$ is a characteristic length scale). As the rate of solidification is completely determined by macrokinetics of diffusion of the solute, its time scale coincides with the relaxation time of solute diffusion and, thus, considerably exceeds the thermal relaxation times. What this means is the temperature field has time enough to follow the varying field of the solute concentration and, thereby, can be regarded as linear.

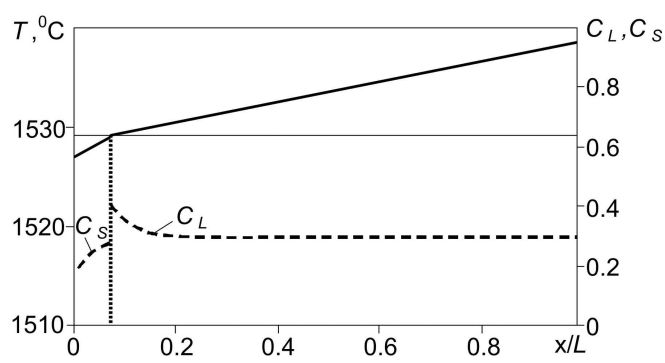

Fig. 1. Temperature (solid lines), impurity concentration (dashed lines) profiles and the front position (vertical line) at $t=150 \mathrm{~s}, X(150)=0.076 L$.

Figure 4 shows that the rate of solidification is a linear function up to the emergence of a mushy layer while the crystallization front moves in accordance with a parabolic law. Increasing the cooling coefficient increases the rate of solidification due to a build up of the heat flux released from the solid wall $x=0$. The latter decreases the time of initiation of a mushy layer. A build up of the temperature gradient $g_{\mathrm{L}}$ (inflowing heat flux passes through the solid wall $x=L$ ) increases $t_{*}$ (see Fig. 5). This is due to the fact that increasing $g_{\mathrm{L}}$ increases the impurity mass, which must be displaced by the growing solid phase to satisfy inequality (10). This process taking much longer corresponds to greater values of the interface coordinate.

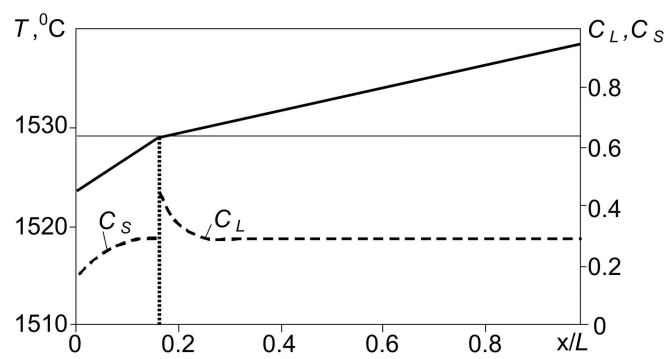

Fig. 2. Temperature (solid lines), impurity concentration (dashed lines) profiles and the front position (vertical line) at $t=t_{*}=221.9 \mathrm{~s}, X(221.9)=0.015 L$.

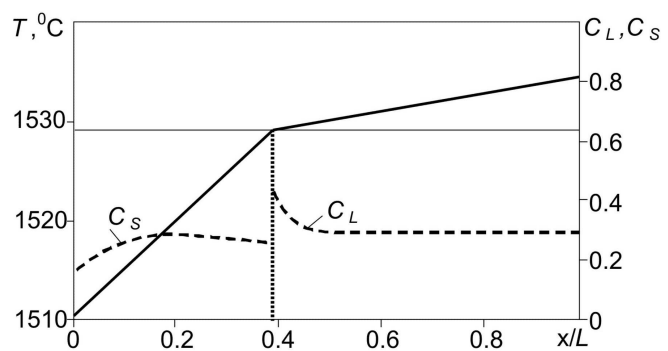

Fig. 3. Temperature (solid lines), impurity concentration (dashed lines) profiles and the front position (vertical line) at $t=360 \mathrm{~s}, X(360)=0.390 L$.

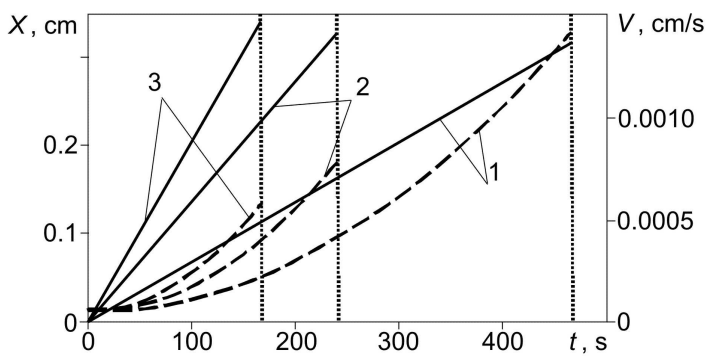

Fig. 4. The interface coordinate $X(t)$ (dashed lines) and the solidification rate $V(t)=\mathrm{d} X / \mathrm{d} t$ (solid lines) as functions of solidification time for different cooling coefficients: $\alpha_{a}=0.01 \mathrm{cal} /\left(\mathrm{s}^{2} \mathrm{~cm}^{2}\right)(1), \alpha_{a}=$ $0.02 \mathrm{cal} /\left(\mathrm{s}^{2} \mathrm{~cm}^{2}\right)(2), \alpha_{a}=0.03 \mathrm{cal} /\left(\mathrm{s}^{2} \mathrm{~cm}^{2}\right)(3)$.

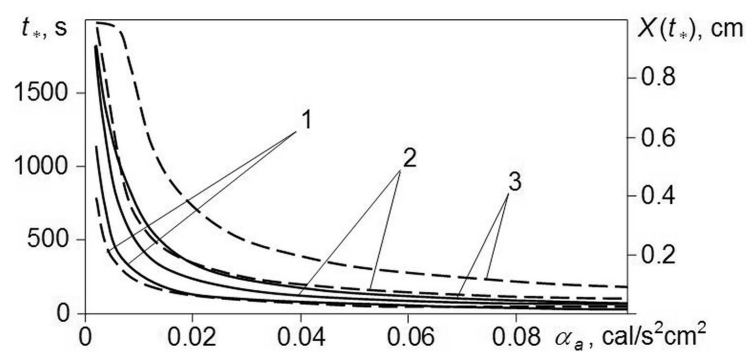

Fig. 5. The time and the corresponding value of front position of the emergence of a mushy layer as functions of the cooling coefficient for different temperature gradients: $g_{\mathrm{L}}=5^{\circ} \mathrm{C} / \mathrm{cm}(1), g_{\mathrm{L}}=10^{\circ} \mathrm{C} / \mathrm{cm}(2)$, $g_{\mathrm{L}}=15^{\circ} \mathrm{C} / \mathrm{cm}(3)$. 


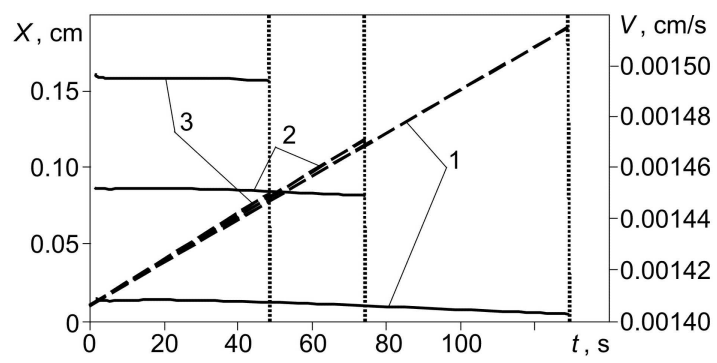

Fig. 6. The interface coordinate $X(t)$ (dashed lines) and the solidification rate $V(t)=\mathrm{d} X / \mathrm{d} t$ (solid lines) as functions of solidification time for different cooling coefficients: $\alpha_{p}=0.0039 \mathrm{cal} /\left(\mathrm{cm}^{2} \mathrm{~s}^{\circ} \mathrm{C}\right)(1), \alpha_{p}=$ $0.0040 \mathrm{cal} /\left(\mathrm{cm}^{2} \mathrm{~s}^{\circ} \mathrm{C}\right)(2), \alpha_{p}=0.0041 \mathrm{cal} /\left(\mathrm{cm}^{2} \mathrm{~s}^{\circ} \mathrm{C}\right)(3)$, $T_{E}=20^{\circ} \mathrm{C}$.

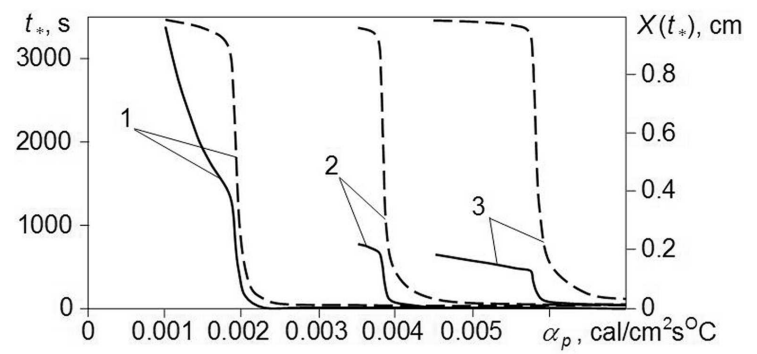

Fig. 7. The time and the corresponding value of front position of the emergence of a mushy layer as functions of the cooling coefficient for different temperature gradients: $g_{\mathrm{L}}=5^{\circ} \mathrm{C} / \mathrm{cm}(1), g_{\mathrm{L}}=10^{\circ} \mathrm{C} / \mathrm{cm}(2)$, $g_{\mathrm{L}}=15^{\circ} \mathrm{C} / \mathrm{cm}(3), T_{E}=20^{\circ} \mathrm{C}$.

Let us especially emphasize that the aforementioned problem frequently met in metallurgy and geophysics can be formulated with different boundary conditions at the solid walls (see, among others [7-9]). As an example, let us consider the case of natural cooling of the solid wall $x=0$. In such a situation, the boundary condition (5) must be replaced by the following condition at the solid wall:

$$
k_{\mathrm{S}} \frac{\partial T_{\mathrm{S}}}{\partial x}=\alpha_{p}\left(T_{\mathrm{S}}-T_{E}\right), \quad x=0,
$$

where $\alpha_{p}$ and $T_{E}$ stand for the cooling coefficient and the external temperature. A nonlinear dynamics of so- lidification in this case is illustrated in Figs. 6 and 7 . Thus, for example, the rate of solidification decreases as the process time increases due to the fact that an ingot mould can cool down at room temperature $\left(T_{E}=20^{\circ} \mathrm{C}\right)$. From the physical point of view the role of physical and operating parameters for this cooling regime is analogous to the active regime considered above.

Let us emphasize in conclusion that the time of initiation of a mushy layer depends on different solidification parameters and, in principle, can be increased varying these parameters. The latter enables us to control the ingot structure (smooth, cellular, dendritic etc.) because, as a rule, a mushy layer is responsible for the evolution of some growing structures leading to different inhomogeneities and properties of solid materials.

\section{Acknowledgments}

This work was made possible due to the financial support of grants Nos. 08-01-00298, 09-08-00844, 07-0396069 Ural, 07-01-96091 Ural (Russian Foundation for Basic Research), MD-4563.2008.2. (President Grant) and 2.1.1/2571 (Ministry of Education).

\section{References}

[1] G.P. Ivantsov, Dokl. Akad. Nauk SSSR 81, 179 (1947).

[2] R.N. Hills, D.E. Loper, P.H. Roberts, Quantum J. Appl. Math. 36, 505 (1983).

[3] D.V. Alexandrov, A.P. Malygin, Int. J. Heat Mass Transfer 49, 763 (2006).

[4] D.L. Aseev, D.V. Alexandrov, Int. J. Heat Mass Transfer 49, 4903 (2006).

[5] Yu.A. Buyevich, D.V. Alexandrov, V.V. Mansurov, Macrokinetics of Crystallization, Begell House, New York 2001.

[6] A.A. Samarski, Theory of Difference Schemes, Nauka, Moscow 1989

[7] P.K. Galenko, O. Fanke, J. Wang, D.M. Herlach, Mater. Sci. Eng. A 375-377, 488 (2004).

[8] D.V. Alexandrov, A.P. Malygin, I.V. Alexandrova, Ann. Glaciol. 44, 118 (2006).

[9] D.V. Alexandrov, I.G. Nizovtseva, Dokl. Earth Sci. 419, 359 (2008). 